\title{
The Google Earth Engine Digitisation Tool (GEEDiT) and the Margin change Quantification Tool (MaQiT) - simple tools for the rapid mapping and quantification of changing Earth surface margins
}

\author{
James M. Lea \\ Department of Geography annd Planning, School of Environmental Sciences, University of Liverpool, \\ Liverpool, L69 7ZT, UK \\ Correspondence: James M. Lea (j.lea@liverpool.ac.uk)
}

Received: 11 March 2018 - Discussion started: 22 March 2018

Revised: 4 June 2018 - Accepted: 4 July 2018 - Published: 13 July 2018

\begin{abstract}
Changes in margins derived from satellite imagery are quantitative indicators of the environmental processes and drivers acting on the Earth's surface, for example retreating ice margins or coastal changes with rising sea level. However, the large-scale rapid visualisation and analysis of the satellite record is often impractical due to factors such as computer processing power, software availability, internet connection speed and/or user expertise in remote sensing. Here are presented three new, freely accessible tools that together can be used to process, visualise and review data from the full Landsat 4-8 and Sentinel 1-2 satellite records in seconds, enabling efficient mapping (through manual digitisation) and automated quantification of margin changes. These tools are highly accessible for users from a range of remote-sensing expertise (from academics to high school students), with minimal computational, licensing and knowledge-based barriers to access. The Google Earth Engine Digitisation Tool (GEEDiT) allows users to define a point anywhere on the planet and filter data from each satellite for user-defined time frames, maximum acceptable cloud cover extent, and options of predefined or custom image band combinations via a simple graphical user interface (GUI). GEEDiT allows georeferenced vectors to be easily and rapidly mapped from each image, with image metadata and user notes automatically appended to each vector, which can then be exported for subsequent analysis. The GEEDiT Reviewer tool allows users to quality control their own/others' data and also filter existing datasets based on the spatial/temporal requirements for their particular research question. The Margin change Quantification Tool (MaQiT) is complementary to GEEDiT and GEEDiT Reviewer, allowing the rapid quantification of these margin changes by utilising two well-established methods that have previously been used to measure glacier margin change and two new methods via a similarly simple GUI. A case study of the lake-terminating glacier Breiðamerkurjökull, Iceland, is used to demonstrate the complementary functionality of GEEDiT, GEEDiT Reviewer and MaQiT, though it should be noted that MaQiT is also suitable for the (re-)analysis of existing datasets not generated by GEEDiT. MaQiT has been developed with the original aim of quantifying tidewater glacier terminus change, though the methods included within the tool have potential for wide applications in multiple areas of Earth surface science (e.g. coastal and vegetation extent change). It is hoped that these tools will allow a wide range of researchers and students across the geosciences to efficiently map, analyse and access volumes of data that would have previously proven prohibitive.
\end{abstract}




\section{Introduction}

Satellite data provide an invaluable record of spatial and temporal change on the Earth's surface. However, the volume and scale of data available for analysis (coupled with computational, software licensing, data storage, internet connectivity and knowledge-based barriers to entry) mean that users may require a significant amount of time to go from downloading an image to finalising its analysis. This can be exemplified in the study of tidewater glacier calving margins where a large volume of remote-sensing imagery exists, though spatially large-scale studies are often required to focus on a number of census time frames (e.g. Cook et al., 2005; Moon and Joughin, 2008; Carr et al., 2017; Bunce et al., 2018), while detailed studies often focus on a relatively small number of sites (e.g. Bevan et al., 2012; Motyka et al., 2017).

The availability of satellite imagery via application programming interfaces (APIs) and increasingly via platforms such as Google Earth Engine (Gorelick et al., 2017), Sentinel Hub's Earth Observation Explorer (Sinergise, 2018) and Planet (Planet Labs Inc., 2018) means that these data are becoming increasingly accessible. However, the ability of users to access these data at such a large scale is currently limited by the need for either knowledge of coding and/or for downloading, storage and processing of substantial volumes of data. Even where users are comfortable with such requirements, images may still prove time-consuming to effectively visualise and finally analyse, thus taking further time. This can severely limit the scale of analysis that it is practical for researchers to undertake.

The identification of temporally evolving margins digitised from this imagery is frequently used across Earth surface sciences to provide key temporal and/or spatial insight into the system of interest (e.g. Kuenzer et al., 2014; Roelfsema et al., 2013; Fitzpatrick et al., 2014; Lynch et al., 2016). Although different geoscientific problems will have different temporal and spatial data coverage requirements, a user's ability to map margins accurately will depend on the effective visualisation of imagery, while generating temporally detailed datasets is dependent on achieving this efficiently and consistently for a large number of images. However, even if a substantial volume of margin change data can be generated, a subsequent issue is the rapid and accurate quantification of these changes.

This study presents three simple-to-use tools that when used together significantly improve the efficiency of visualising and exploring satellite imagery, while also allowing the rapid mapping and quantification margin changes directly from them. The first is the Google Earth Engine Digitisation Tool (GEEDiT), which allows the rapid visualisation, mapping and export of digitised margins without the need to download imagery to the user's computer. It is also possible to use GEEDiT to map multiple features directly from an individual image and append notes to individual margins and images. The second is the GEEDiT Reviewer tool that allows data previously generated by GEEDiT to be quality controlled and filtered. The third is the Margin change Quantification Tool (MaQiT) that allows the rapid quantification of these digitised margin changes, utilising two existing methods (Lea et al., 2014) and two new methods that have commonly been used in the quantification of tidewater glacier margin change. Although initially developed for glaciological applications, each of these quantification methods are likely to have applications in the quantification of margin change in other areas of Earth surface sciences such as coastal change, lake level evolution, and vegetation and urban extent change, amongst others.

\section{Google Earth Engine Digitisation Tool (GEEDiT) and GEEDiT Reviewer}

GEEDiT and GEEDiT Reviewer are written in JavaScript within Google Earth Engine's (GEE) API (Gorelick et al., 2017). GEEDiT is designed to allow satellite imagery from Landsat 4-8 and Sentinel 1-2 to be visualised rapidly within a standard web browser, also allowing the digitisation and export of polyline and/or polygon vector data in GeoJSON (Georeferenced JavaScript Object Notation format) or KML/KMZ (Keyhole Markup Language/Keyhole Markup Zipped format compatible with Google Earth) formats. GEE does not currently support the export of data in shapefile format, though a tool is included within MaQiT to both merge and convert GeoJSON files to a single shapefile (see Sect. 3). This means that data digitised during multiple GEEDiT sessions can be merged and/or converted for use either in MaQiT or a traditional geographic information system (GIS) platform.

The GEEDiT Reviewer tool is separate to GEEDiT, allowing users to review existing datasets generated by GEEDiT. It can be used to both quality control their own and others' data against the original imagery used for margin digitisation and filter datasets based on the temporal requirements of their research question. To use GEEDiT Reviewer, the data generated needs to be uploaded to GEE as an "asset" (see the Supplement readme file). Users also have the option to make assets publicly accessible via the privacy options associated with each file within GEE.

The tools have been developed and tested in Google Chrome though should also function in other widely used browsers such as Internet Explorer, Mozilla Firefox and Safari.

Access to GEE for research, education and non-profit use is free of charge, though users are required to register for access (https://signup.earthengine.google.com/). The only other requirement is access to Google Drive (included as part of signing up to a Gmail email address), which is also free. The tool can be run and used by following the steps outlined in the supplementary readme file appended to this article. 
Table 1. Description of satellites and optional band combinations that are built into GEEDiT. Note that certain user-defined custom band combinations may have lower resolution.

\begin{tabular}{|c|c|c|c|c|c|c|}
\hline Satellite & $\begin{array}{l}\text { Imagery } \\
\text { type }\end{array}$ & Lifespan & $\begin{array}{r}\text { True colour } \\
\text { bands (R-G-B) }\end{array}$ & $\begin{array}{r}\text { False colour } \\
\text { bands (R-G-B) }\end{array}$ & $\begin{array}{r}\text { Image } \\
\text { resolution } \\
(\mathrm{m})\end{array}$ & Notes \\
\hline Landsat 4 & Optical & Jul 1982-Dec 1993 & $3-2-1$ & $5-4-3$ & 30 & Gamma: 2 \\
\hline Landsat 5 & Optical & Mar 1984-Jan 2013 & $3-2-1$ & $5-4-3$ & 30 & Gamma: 2 \\
\hline Landsat 7 & Optical & Apr 1999- & $3-2-1$ & $5-4-3$ & 15 & $\begin{array}{l}\text { Pansharpened from } 30 \text { to } 15 \mathrm{~m} \text { using } \\
\text { band } 8 \text {; scan line corrector failure } \\
\text { after } 31 \text { May 2003; Gamma: } 2\end{array}$ \\
\hline Landsat 8 & Optical & Feb 2013- & $4-3-2$ & $6-5-4$ & 15 & $\begin{array}{l}\text { Pansharpened from } 30 \text { to } 15 \mathrm{~m} \\
\text { using band } 8 \text {; Gamma: } 2\end{array}$ \\
\hline $\begin{array}{l}\text { Sentinel } 1 \mathrm{~A} \\
\text { and } 1 \mathrm{~B}\end{array}$ & SAR & $\begin{array}{l}\text { 1A - Apr 2014- } \\
\text { 1B - Apr 2016- }\end{array}$ & - & - & 10 & $\begin{array}{l}\text { Horiz. transmit/horiz. receive }(\mathrm{HH}) \text {, } \\
\text { or vert. transmit/vert. receive }(\mathrm{VV}) \text {; } \\
\text { Min.: }-20 \text {; max.: } 1\end{array}$ \\
\hline $\begin{array}{l}\text { Sentinel 2A } \\
\text { and 2B }\end{array}$ & Optical & $\begin{array}{l}\text { 2A - Jun 2015- } \\
\text { 2B - Mar 2017- }\end{array}$ & $4-3-2$ & $8-4-3$ & 10 & $\begin{array}{l}\text { Gamma: } 2 \text {; } \\
\text { gain: } 0.025\end{array}$ \\
\hline
\end{tabular}

Band combinations, gamma options, max./min. ranges and opacity can be varied manually via the "Layers" tab at the top right of the screen.

Imagery is always stored in "Layer 1".

Video guides that explore the functionality of GEEDiT and GEEDiT Reviewer have also been produced (https://www. youtube.com/channel/UCboaSHUmNaY7eAeScS0B2_Q).

\subsection{Image visualisation}

GEEDiT can visualise imagery from optical imaging platforms as either natural (true colour), false-colour or custom band combinations. Sentinel-1 synthetic aperture radar (SAR) data can also be visualised as grey scale images (Table 1). SAR data exist in either single- or dual-band polarisation bands, though not every band is collected for every scene. To maximise the temporal and spatial coverage for the tool, GEEDiT will visualise whichever single polarisation band is available (either horizontal transmit/horizontal receive $(\mathrm{HH})$ or vertical transmit/vertical receive (VV)) for both ascending and descending orbits for a particular time and location. The polarisation and type of orbit (ascending/descending) of each SAR image are displayed at the top right panel alongside the satellite name, date and image number/total number of images available.

Note that the location accuracy of a feature identified in Sentinel 1 imagery can be significantly degraded in areas that have undergone several metres of topographic change relative to the digital elevation model used for terrain correction (SRTM 30 for areas $<60^{\circ}$ latitude; otherwise ASTER DEM). The scale of geolocation error will also vary depending on whether the image was acquired during an ascending or descending orbit (see Sect. 4). Care should therefore be taken in using Sentinel 1 data in such scenarios (e.g. where significant surface thinning of a glacier/ice sheet has occurred). Qualitative checks for this can be achieved by a comparison of Sentinel 1 data with optical satellite data acquired at a similar time.
A summary of the default parameters used to visualise both the optical and SAR imagery is given in Table 1. Further information regarding each satellite image collection can be obtained by searching for it in the search bar at the top of the GEE interface.

\subsection{Output of margin/boundary data}

Vector data are output by GEEDiT in decimal degrees format so as to be easily read by GIS software and/or subsequently converted to different spatial projections. Key metadata that link each margin to information about the image it has been digitised from are appended to each digitised line (see the Supplement readme). This includes each image's unique path identifier, meaning that results generated by GEEDiT are directly traceable back to its original image. This can be achieved using GEEDiT Reviewer, where existing datasets can be quality controlled and filtered. If it is anticipated that the data digitised in GEEDiT will be analysed subsequently in a different GIS environment, it is recommended that data are output as GeoJSON files (this is the default option), since these can be merged/converted to shapefile format using MaQiT. Note that KML/KMZ files do not always allow metadata to be retained when they are imported into standard GIS software packages such as ArcGIS and QGIS using "out of the box" tools. Exporting data from GEEDiT/GEEDiT Reviewer in KML/KMZ formats therefore may make subsequent analysis problematic.

\section{Margin change Quantification Tool - MaQiT}

MaQiT has been produced to rapidly quantify margin change for use in subsequent analysis (outputs provided as Excel/OpenOffice compatible csv spreadsheets and as initial 
plots generated by the tool) and also convert and merge single/multiple GeoJSON/shapefile files into a single shapefile. Although MaQiT uses methods that have been developed for the quantification of tidewater glacier margin change (e.g. Lea et al., 2014), they will be transferable to tracking margin changes in other environments. Each quantification method has its own benefits and pitfalls, meaning that appropriate method selection should be based primarily on the research question being asked.

\subsection{Installing/running MaQiT}

Although MaQiT was written in Matlab ${ }^{\circledR}$, its code has been compiled into a standalone application (installers available for Windows and Mac) meaning that it can be installed and run by users without a Matlab ${ }^{\circledR}$ license and free of any charges. The only prerequisite for this is to download the free software, Matlab ${ }^{\circledR}$ Runtime, though users should be prompted once to do this automatically the first time the installer is opened. Users do not require a general Matlab ${ }^{\circledR}$ license to run MaQiT.

If users do have a general Matlab ${ }^{\circledR}$ license, MaQiT can also be run by copying all the scripts to a single directory and running the MaQiT.m script. This will open MaQiT's graphical user interface (GUI), allowing it to be used in a similar manner to the standalone application (see Supplement readme). The methods used by MaQiT can also be run programmatically as Matlab ${ }^{\circledR}$ functions. Where multiple datasets from large numbers of sites exist, this provides the potential for large-scale rapid analysis. The results generated after the analysis of each location can be accessed via a data structure named "Results" in the Matlab ${ }^{\circledR}$ workspace or be written to a csv spreadsheet identical to that produced by the standalone application. MaQiT also makes use of publicly submitted functions obtained from the Mathworks File Exchange (Palacios, 2006; D'Errico, 2012a, b, 2013; Dugge, 2015). Copies of these functions are compiled into the standalone version of MaQiT and are included in the code files appended to this publication. Instructions regarding the required inputs for MaQiT and how to use the tool are included in the Supplement readme file.

\subsection{Methods of quantifying margin/boundary changes in MaQiT}

Four different methods of quantifying margin changes are included in MaQiT, two of which are established approaches that are used in the tracking of tidewater glacier terminus change (e.g. Cook et al., 2005; Lea et al., 2014), while two are new methods designed for the same purpose, though with potential wider applications.

\subsubsection{Centreline method}

This is the simplest approach to tracking margin change, measuring the linear distance along a centreline between two boundaries (e.g. Cook et al., 2005; VanLooy and Forster, 2008; Fig. 1a). This approach provides a one-dimensional measure of change that does not account for the behaviour of the entire margin; only the point of intersection between the centreline and the margin (Lea et al., 2014). While this method is simple, the method is best suited to scenarios/research questions where it can be assumed that the margin is uniformly advancing/retreating or the area of the margin that is of interest is narrow (i.e. a few pixels across). If either of these assumptions are not valid, or a higher level of detail is required, then an alternative method of tracking change would be more suitable.

\subsubsection{Curvilinear box method}

This method provides a linear measure of margin advance/retreat by defining a box of fixed width spanning the centreline that intersects with the margin, before dividing the area of this box by its width (Lea et al., 2014; Fig. 1b). The user is required to define the box width. The result provides the one-dimensional distance from the start of a centreline to the mean location of the part of the margin that intersects with the box. This method provides a more representative width-averaged value of marginal change compared to the centreline method. The method is an extension of the box method used by Moon and Joughin (2008) but has the advantage that the defined box does not need to be rectilinear (i.e. it allows the box to follow potentially non-linear topographic features such as fjords/valleys).

If the defined box width is wider than the margin itself/one or more edges of the box do not intersect with the margin, the box will be "closed" by lines that take the shortest distance from the start/end points of the margin to the box edge. If this scenario is a possibility (i.e. if the box width is greater than that of the margin width), it is important that the centreline used extends upstream and downstream of the margins for a greater distance than the shortest path between the centreline and the start/end points of any of the digitised margins (i.e. the centreline should extend up/downstream for more than twice the width of the longest margin). Failure to do this may result in errors in the geometry of the boxes used to obtain measurements. This can be checked visually using the "Plot output" option in MaQiT, which shows the geometries of each box that is used to quantify margin change. If errors of this nature do occur, it is recommended that the user redraws the centreline using GEEDiT Reviewer, extending the start/end points of the centreline further up/downstream.

Although this method has the potential to account for a higher proportion of the margin than the centreline method, it will not account for the entire margin. It is therefore suit- 

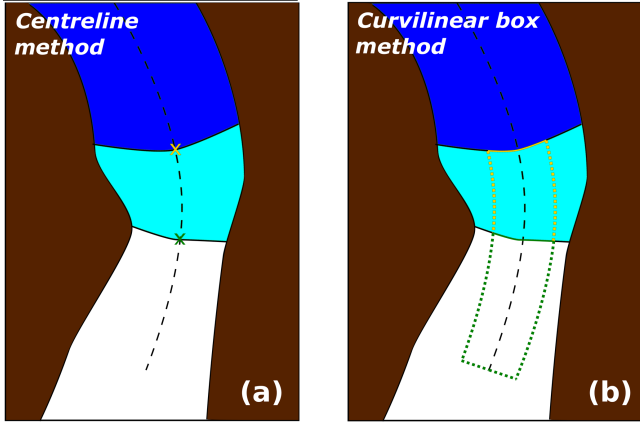

Variable box

method
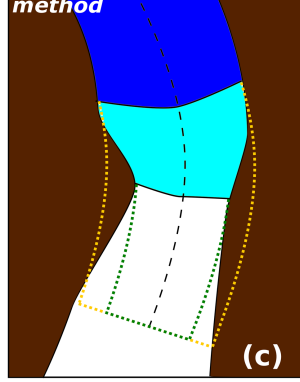

Multi-centreline

method

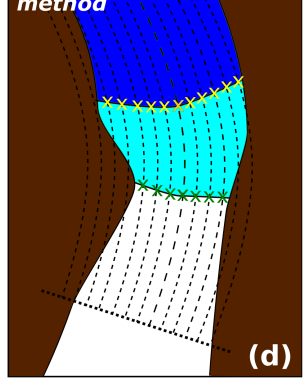

Figure 1. Methods of margin change quantification that can be applied in MaQiT. Example shows the retreat of a tidewater glacier with ice (white), the former glacier extent (light blue) and open water (dark blue). (a) The centreline method takes the linear distance from the start of the centreline to the first point of intersection between the centreline and the margin. (b) The curvilinear box method generates a box of a user-defined fixed width that is closed at its downstream edge by the digitised margin, with a one-dimensional measure of the distance from the start of the centreline obtained by dividing the box area by the box width (note that yellow box margin also extends to the start of the centreline). (c) The variable box method operates on the same principle as the curvilinear box method, though box width is automatically defined by MaQiT as the total distance from the end nodes to the centreline. (d) The multicentreline method operates on the same principle as the centreline method, though multiple, regularly spaced lines are used to build a two-dimensional representation of margin change, with the output using a colour scale to visualise distance.

able to apply if the user is interested in obtaining an averaged measure of change for a particular section of the margin.

\subsubsection{Variable box method}

This method is similar to the curvilinear box method, though instead of using a fixed box width, it uses the full width of the margin (Fig. 1c). The width of each box is defined as the total distance between the start and end nodes of the margin. This allows a one-dimensional distance of change to be determined that includes the full extent of the digitised margin. This has the advantage of accounting for all margin information available, though similar caveats apply to this method as the curvilinear box method.
To ensure the accuracy of results given by this method, it is important that the start/end points of each margin are at physically meaningful locations. To ensure the comparability of results, this is especially important where it is possible that the margin will have occupied a given location more than once. An example of this would be a tidewater glacier, with physically meaningful start/end points being the two points at which the glacier margin, sea and land meet (i.e. the distance between the start and end points of the margin would give an accurate measurement of glacier width). If only part of the ice front was digitised, then the method would give an inaccurate result that may not be comparable to subsequent observations. Where the method is applied using arbitrarily/semi-arbitrarily defined start/end points then the variable box method may over-/under-predict extent depending on how much of and what parts of the margin have or have not been digitised.

\subsubsection{Multi-centreline method}

This method extends the centreline method to include multiple centrelines that span the width of a margin. This results in many one-dimensional measures of change across the entire margin width, thus allowing the spatial variability in margin advance/retreat to be quantified (Fig. 1d). MaQiT visualises the distance changes that occur as colour change on an $x-y$ plot (see Sect. 4). Where the process of interest may occur over timescales longer than the intervals between observations, it is also possible to define the temporal "window" over which margin changes will be quantified that will improve data visualisation. For example, if a margin observation exists every 8 days but the research question requires comparison of observations made between 30 to 40 days apart, this can optionally be defined and MaQiT will automatically filter the observations. These results can also be output to csv files as time-distance tables.

\subsection{Viewing results from MaQiT}

The results generated by MaQiT for each method can be visualised as a series of plots that are automatically generated by the tool. Due to the nature of each method, the plots used to visualise the results vary between methods (i.e. the centreline method does not include a plot to check box geometry as it does not require using a box). For the centreline and curvilinear and variable box methods, there are either three or four plots shown (e.g. Supplement; Figs. S1-S4). The first plot shows all the margins to allow the user to check that they have been read in correctly by MaQiT. The second plot is only included for the curvilinear and variable box methods as it allows the user to check that the box geometries have been constructed correctly and whether redrawing of the centreline is necessary. The third plot shows a time series of distance change of the margin. The multi-centreline method provides a different output, showing results as a se- 
Table 2. Description of bands for optical imagery satellites.

\begin{tabular}{|c|c|c|c|c|c|c|c|c|}
\hline \multirow[b]{2}{*}{$\begin{array}{l}\text { Band } \\
\text { number }\end{array}$} & \multicolumn{2}{|c|}{ Landsat 4 and 5} & \multicolumn{2}{|c|}{ Landsat 7} & \multicolumn{2}{|c|}{ Landsat 8} & \multicolumn{2}{|l|}{ Sentinel 2} \\
\hline & $\begin{array}{l}\text { Band } \\
\text { description }\end{array}$ & $\begin{array}{r}\text { Resolution } \\
(\mathrm{m})\end{array}$ & $\begin{array}{l}\text { Band } \\
\text { description }\end{array}$ & $\begin{array}{r}\text { Resolution } \\
(\mathrm{m})\end{array}$ & $\begin{array}{l}\text { Band } \\
\text { description }\end{array}$ & $\begin{array}{r}\text { Resolution } \\
(\mathrm{m})\end{array}$ & $\begin{array}{l}\text { Band } \\
\text { description }\end{array}$ & $\begin{array}{r}\text { Resolution } \\
\text { (m) }\end{array}$ \\
\hline 1 & Blue & 30 & Blue & 30 & Ultra blue & 30 & Coastal aerosol & 60 \\
\hline 2 & Green & 30 & Green & 30 & Blue & 30 & Blue & 10 \\
\hline 3 & Red & 30 & Red & 30 & Green & 30 & Green & 10 \\
\hline 4 & Near-IR & 30 & Near-IR & 30 & Red & 30 & Red & 10 \\
\hline 5 & Shortwave-IR 1 & 30 & Shortwave-IR 1 & 30 & Near-IR & 30 & Vegetation red edge & 20 \\
\hline 6 & Thermal & $120^{*}(30)$ & Thermal & $60 *(30)$ & Shortwave-IR 1 & 30 & Vegetation red edge & 20 \\
\hline 7 & Shortwave-IR 2 & 30 & Shortwave-IR 2 & 30 & Shortwave-IR 2 & 30 & Vegetation red edge & 20 \\
\hline 8 & - & - & Panchromatic & 15 & Panchromatic & 15 & Near-IR & 10 \\
\hline $8 \mathrm{~A}$ & - & - & - & - & - & - & Narrow near-IR & 20 \\
\hline 9 & - & - & - & - & Cirrus & 30 & Water vapour & 60 \\
\hline 10 & - & - & - & - & Thermal-IR 1 & $100^{*}(30)$ & Shortwave-IR - cirrus & 60 \\
\hline 11 & - & - & - & - & Thermal-IR 2 & $100 *(30)$ & Shortwave-IR & 20 \\
\hline 12 & - & - & - & - & - & - & Shortwave-IR & 20 \\
\hline
\end{tabular}

* indicates where data have been resampled to the resolution given in brackets.

Table 3. Summary statistics for the margins digitised from different satellites.

\begin{tabular}{lrrrrr}
\hline Satellite & $\begin{array}{r}\text { Margins } \\
\text { digitised }\end{array}$ & $\begin{array}{r}\text { Mean path } \\
\text { length (m) }\end{array}$ & $\begin{array}{r}\text { Mean } \\
\text { width (m) }\end{array}$ & $\begin{array}{r}\text { Mean number } \\
\text { of vertices }\end{array}$ & $\begin{array}{r}\text { Mean distance } \\
\text { between points (m) }\end{array}$ \\
\hline Sentinel 1 (asc.) & 39 & 5643 & 3357 & 70.9 & 82.7 \\
Sentinel 1 (desc.) & 39 & 6204 & 3316 & 67.3 & 95.6 \\
Landsat 8 & 38 & 4797 & 3052 & 61.6 & 79.7 \\
Sentinel 2 & 17 & 4644 & 2924 & 64.1 & 77.2 \\
\hline Total & 133 & 5869 & 3203 & 66.6 & 91.1 \\
\hline
\end{tabular}

ries of four rows of plots that show (1) marginal change including every available observation; (2) marginal change using the defined temporal window (if a temporal window is not defined, this plot will be identical to the first plot); (3) absolute distance change between observations from one margin to the next observation; and (4) rate of margin change between observations (e.g. Fig. 5b). The left column of plots shows changes occurring for the entire margin width, while, for reference, the right column shows the one-dimensional results that would otherwise be generated by the centreline method.

It is strongly recommended for all methods that users view results generated by MaQiT as a quality control measure of both the user's data and the successful execution of the analysis.

Users with a standalone MaQiT installation are able to output results to a csv file for subsequent analysis. Values output include year, month, date, serial date (i.e. number of days since January 0th $0000 \mathrm{AD}$ ), margin position on flow line, margin position relative to the most retreated position, margin change compared to previous observation, rate of change from previous observation, margin width, and (for box methods only) box widths and box area. Users with a Matlab ${ }^{\circledR}$ license are able to interrogate and subsequently analyse output via the Results data structure that is generated and located in the workspace and/or export data to a csv file. Note that the multi-centreline method outputs data as three csv files showing margin position, margin change from the previous observation and rate of margin change from the previous observation.

\section{Case study - margin change at Breiðamerkurjökull, Iceland}

Breiðamerkurjökull, SE Iceland $\left(64.11^{\circ} \mathrm{N}, 16.22^{\circ} \mathrm{W}\right)$, is an outlet glacier of the Vatnajökull ice cap that drains into the tidal lagoon Jökulsárlón (Fig. 2). The calving margin of the glacier was digitised at monthly intervals (where possible) for each of Landsat 8, Sentinel 2 and Sentinel 1 (both ascending and descending orbits) for January 2014 to January 2018. This allows a broad intercomparison of any systematic biases that may exist between these platforms in an area that has undergone significant elevation change relative to the digital elevation model (DEM) used for terrain correction of the imagery (Björnsson et al., 2001). A total of 587 images were viewed during digitisation, with 133 ice fronts digitised in to- 
Table 4. MaQiT performance metrics.

\begin{tabular}{llrrr}
\hline Method & Satellite & $\begin{array}{r}\text { Number of } \\
\text { observations }\end{array}$ & $\begin{array}{r}\text { Total calculation } \\
\text { time (s) }\end{array}$ & $\begin{array}{r}\text { Calculation time } \\
\text { per observation (s) }\end{array}$ \\
\hline Centreline method & Landsat 8 & 38 & 0.49 & 0.013 \\
Curvilinear box method & Landsat 8 & 38 & 3.43 & 0.090 \\
Variable box method & Landsat 8 & 38 & 2.81 & 0.074 \\
Multi-centreline method & Landsat 8 & 38 & 4.56 & 0.12 \\
\hline
\end{tabular}

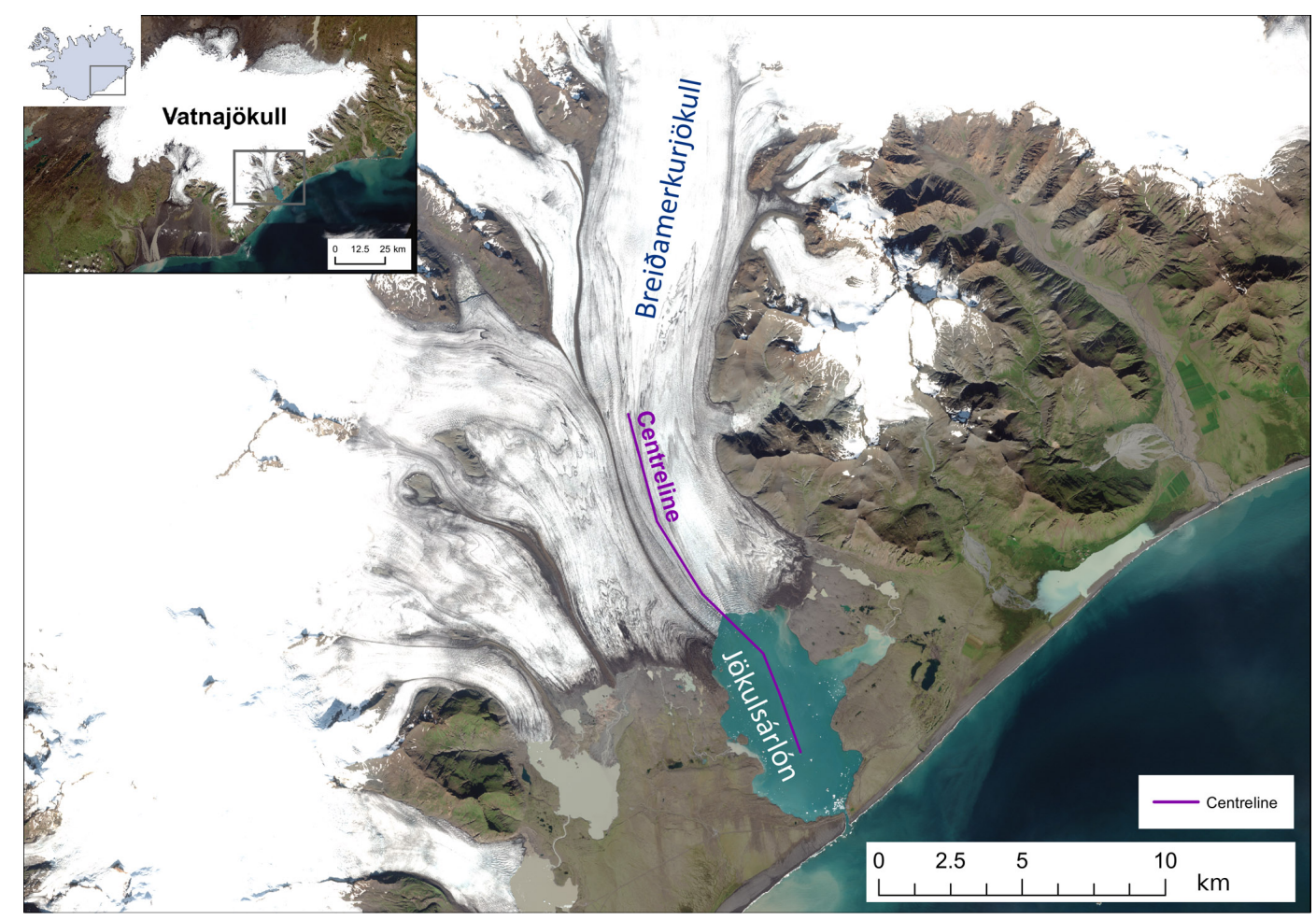

Figure 2. Location map and centreline of Breiðamerkurjökull, SE Iceland. Imagery shows a true-colour composite of four Sentinel 2A scenes acquired on 20 August 2017.

tal. The summary statistics of the digitised margins are given in Table 3. Visualisation and digitisation of the margins were undertaken in four sessions, taking a total time of $2 \mathrm{~h}$ and 3 min. A metric for the level of detail obtained for a margin is given as part of the Results/csv output by dividing the total length of the margin by the number of points digitised (see mean distance between points, Table 3). Shapefiles of the combined Landsat 8 and Sentinel 2 records of margin change are included as a Supplement as example data.

Once digitisation of the ice margins was complete, MaQiT was used to convert and merge the GeoJSON files generated by GEEDiT to a single shapefile. The appended Supplement data can also be visualised in GEEDiT Reviewer by copy and pasting the path users/Jmleaglacio/Breida_L8_S2 into the tool.

It should be emphasised that the method of margin change quantification that should be used for this type of data is heavily dependent on the research question that the user is seeking to address. The analysis undertaken here is only to provide a demonstration of the methods available in MaQiT.

\subsection{Case study results \\ 4.1.1 Intercomparison of results from different satellites}

The curvilinear box method (width: $2000 \mathrm{~m}$ ) was used to illustrate whether any systematic differences exist between margins digitised from different satellites (Fig. 3). Results show that while similar patterns and magnitudes of change are given for each satellite, margins digitised from Sentinel 1 imagery show clear under- and overestimation of margin extent (relative to Sentinel 2 and Landsat 8 imagery) for descending and ascending orbits respectively. One-to-one matches in results are not expected as image acquisitions for the different satellites did not always fall on the same day, 


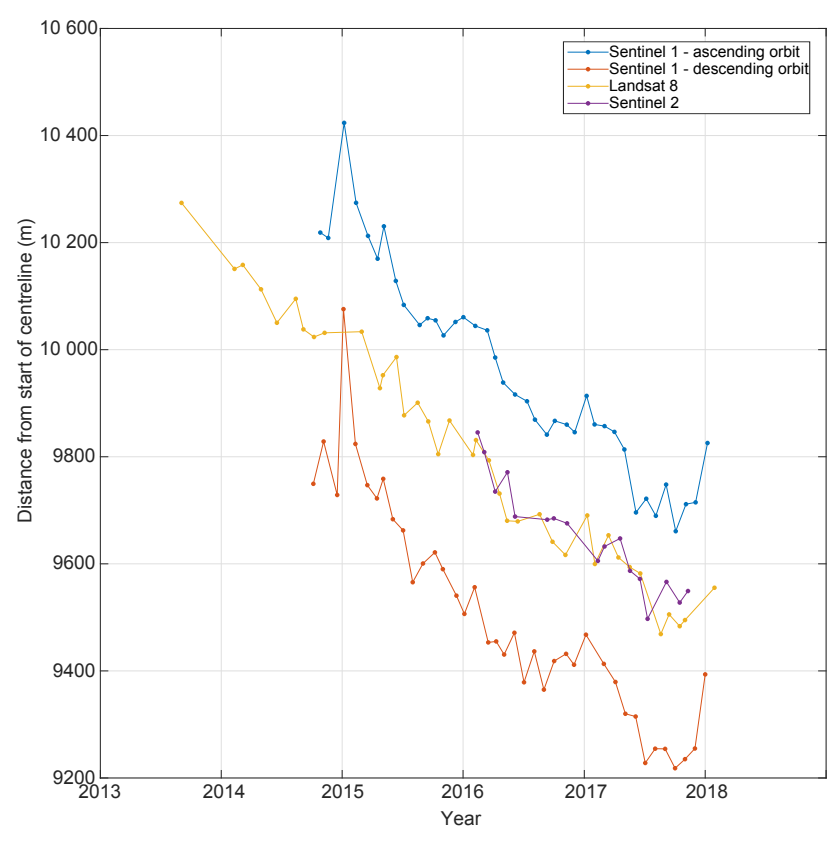

Figure 3. Intercomparison of monthly margin positions at Breiðamerkurjökull given by the curvilinear box method (width: $2000 \mathrm{~m}$ ) digitised from different satellites.

while the margin of Breiðamerkurjökull is known to flow rapidly ( $>5 \mathrm{~m} \mathrm{~d}^{-1}$; Voytenko et al., 2015), meaning that the margin has the potential to be highly dynamic over short timescales (cf. Benn et al., 2017).

Though results from Sentinel 2 and Landsat 8 are broadly comparable, Fig. 3 illustrates that for Sentinel 1 imagery there can be significant mismatch in areas where significant elevation change has occurred relative to the DEM used for initial terrain correction. In environments where considerable elevation change has not occurred the mismatch should be less, though margins from ascending and descending orbits (automatically appended by GEEDiT to margin metadata) should still be checked for systematic biases. Due to the "oblique looking" nature of Sentinel 1 image acquisitions (compared to "vertical looking" for optical satellite imagery), the magnitude of mismatches in areas of elevation change are likely to be variable across an image.

The mismatches shown in these results demonstrate that considerable care should be taken in combining observations from Landsat/Sentinel 2 imagery with Sentinel 1 imagery.

\subsection{Intercomparison of methods for quantifying margin change}

Observations of margin change at Breiðamerkurjökull obtained from Landsat 8 are used to demonstrate the different methods of margin change quantification included in MaQiT.

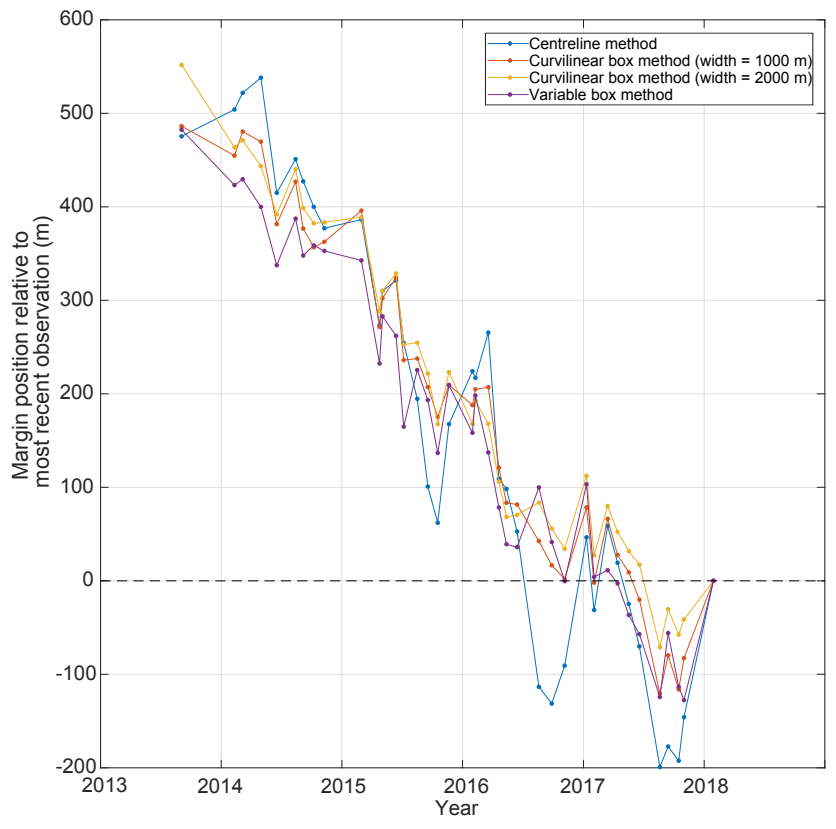

Figure 4. Intercomparison of results from different margin quantification methods applied to the Landsat 8 monthly record of margin positions at Breiðamerkurjökull.

\subsubsection{One-dimensional measures of margin change}

The centreline, curvilinear box and variable box methods provide one-dimensional measures of margin change (i.e. how far advanced/retreated a margin is relative to the distance along a centreline). Figure 4 shows that each of the methods record similar overall patterns of change (i.e. retreat), though at times diverge from each other depending on method/parameter choice. In particular, the centreline method displays a high degree of variability (e.g. 20152018) as it reflects margin change in an extremely localised area. This is in contrast to the other methods that provide results that are more representative of the margin as a whole. It should also be noted that while each method generally agrees on the sign of margin change (i.e. advance or retreat), this is not always the case. In general, methods that account for larger proportions of the margin (i.e. the variable box and curvilinear box method (width: $2000 \mathrm{~m}$ )) are more likely to disagree with methods that account for less of the margin (i.e. centreline and curvilinear box methods (width: $1000 \mathrm{~m}$ )). This highlights the importance of the need to carefully select method/parameter choice with respect to the research question that is being addressed.

\subsubsection{Multi-centreline method}

The multi-centreline method provides a two-dimensional representation of margin change, highlighting regions of the margin that are more susceptible to advance/retreat, in addition to the timing and magnitude of this. It also pro- 


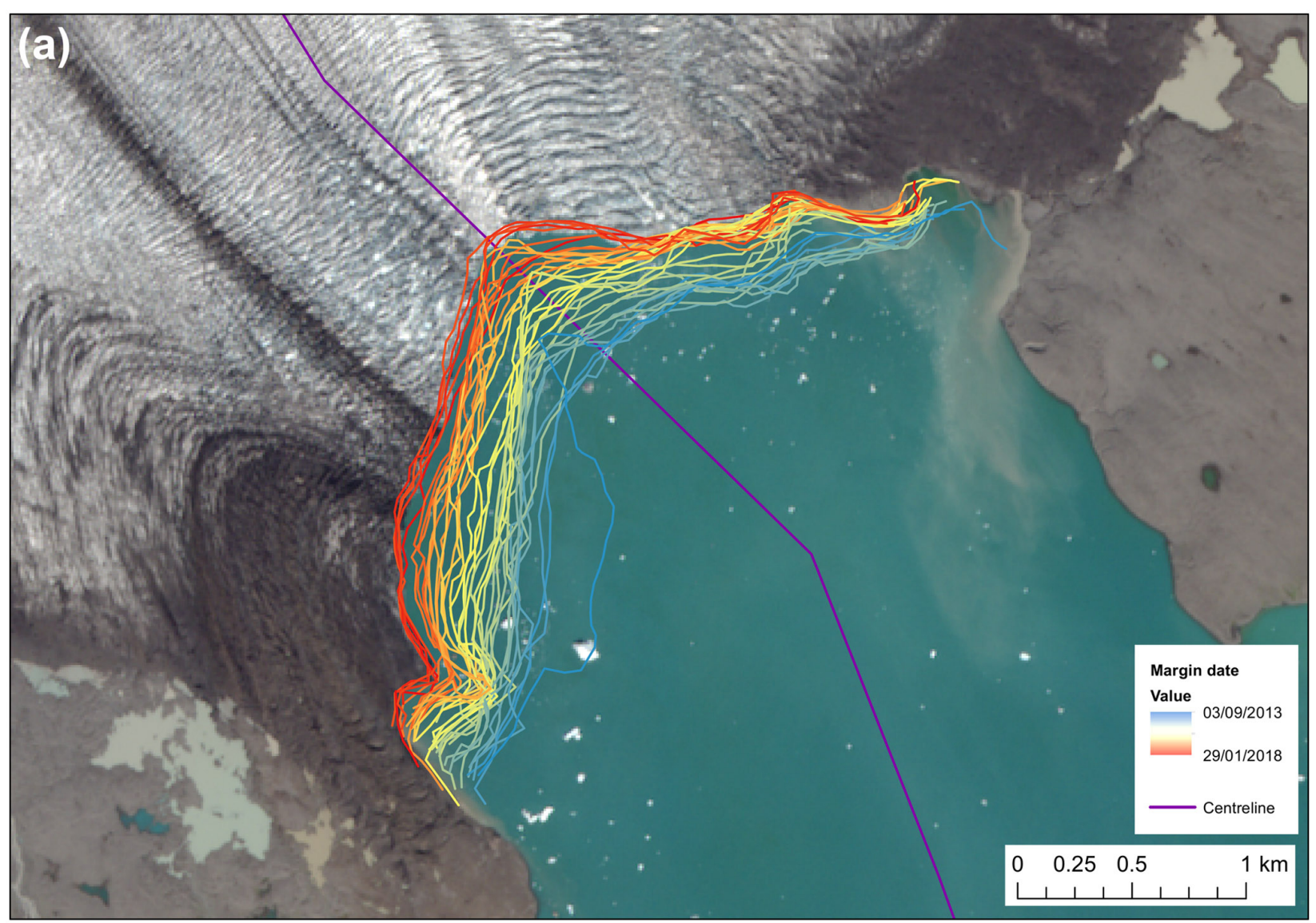

(b)
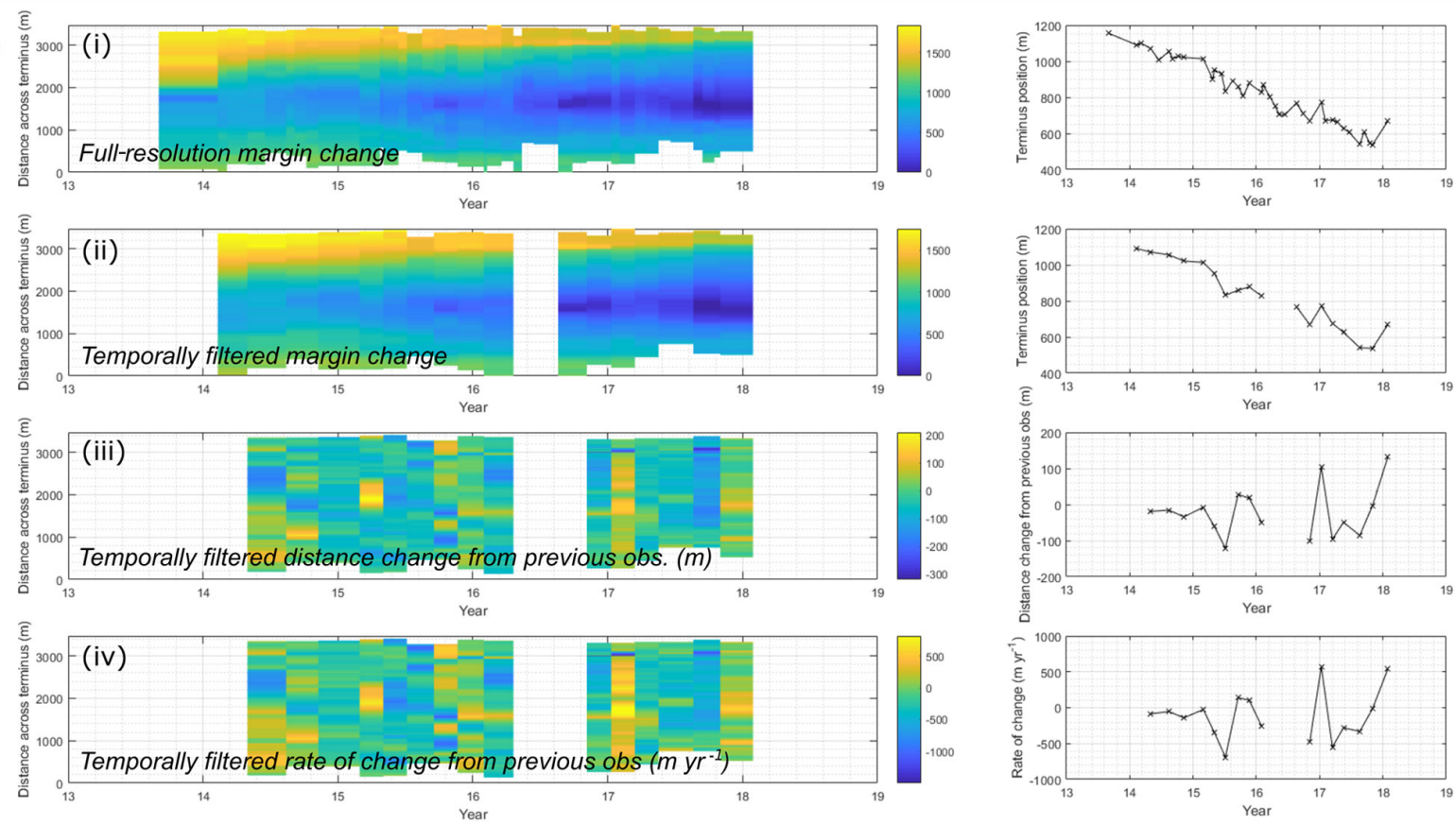

Figure 5. Margin migration for monthly Landsat 8 observations of Breiðamerkurjökull shown as a time series (a) cartographically and (b) as results from the multi-centreline method. Panel (b) has four rows of plots showing (i) the margin position for all available observations relative to the most retreated position across the margin; (ii) margin position observations separated by at least 60 days and a maximum of 120 days (these values are user-defined); (iii) total distance change between observations; and (iv) rate of change of margin in $\mathrm{m} \mathrm{yr}^{-1}$. Right-hand column of plots displays results of the centreline method for comparison. 
vides a means of visualising two-dimensional change as a time series rather than relying on maps of margin change that may otherwise be difficult to interpret in a meaningful way (e.g. Fig. 5a). For the case study, observations were obtained at approximately monthly intervals, though the method has been applied so as to highlight changes over seasonal timescales (60 to 120 days). Results show that the centre of Breiðamerkurjökull's margin is consistently the most retreated (Fig. 5bi, ii) and that there is little seasonal consistency across the entire margin as to whether it advances or retreats and at what rate (Fig. 5biii, iv).

\subsection{MaQiT performance}

Table 4 shows performance metrics of each method from the standalone version MaQiT. The speed at which users would be able to complete comparable analysis without MaQiT is highly dependent on an individual's existing GIS and/or coding competence. However, for those without coding skills and entry level GIS training, it may take a user several minutes to obtain a single value that quantifies the position of one margin. MaQiT, therefore, provides a potentially major improvement in the efficiency with which users can analyse their data. Results produced by MaQiT are also guaranteed to be methodologically consistent and replicable. This makes MaQiT highly suited to the (re-)analysis of repository datasets of margin change.

\section{Summary}

Together GEEDiT, GEEDiT Reviewer and MaQiT provide simple tools for rapid satellite image visualisation, exploration and initial assessment (via notes appended to metadata), digitisation of margins from imagery, review and filtering of existing datasets, and quantification of their changes via multiple methods. They dramatically improve the efficiency with which these analyses can be undertaken and the accessibility of these data to researchers both with and without knowledge of coding. The lack of the requirement to download, process and store imagery on a user's computer, coupled with simple GUIs and no fee-paying licensing requirements also improves the accessibility to these data through the removal of traditional barriers to entry associated with remote sensing and GIS. The tools are therefore suitable for users ranging from high school students to academics.

GEEDiT provides flexibility for the way in which imagery is visualised (i.e. true-colour, false-colour and custom band combinations), while MaQiT gives users the flexibility to rapidly quantify and output measures of margin change. The case study of the calving glacier Breiðamerkurjökull highlights the potential for mismatch between imagery collected via ascending/descending orbits of Sentinel 1 relative to optical imagery satellites such as Landsat and Sentinel 2. Consequently users should take care in combining margin records from Sentinel 1 with those of Landsat/Sentinel 2, especially where significant elevation change may have occurred relative to the DEM that is used for terrain correction of imagery in Google Earth Engine.

Intercomparison of the two existing and two new methods of margin change quantification available in MaQiT illustrate the potential for obtaining potentially substantial differences in margin change values when analysing the same data. This highlights the importance of users selecting the most suitable margin quantification method for their particular research problem. The new multi-centreline method also provides a means of visualising margin change as a time series potentially in a clearer manner than is possible cartographically. While these techniques have predominantly been developed for the quantification of tidewater glacier margin change, they could also be useful for researchers investigating coastal change, dune migration and vegetation extent changes amongst other areas of Earth surface science.

Data availability. Links to GEEDiT and GEEDiT Reviewer and downloads for MaQiT can be found at the following website: www. liverpoolGEE.wordpress.com.

\section{The Supplement related to this article is available online at https://doi.org/10.5194/esurf-6-551-2018-supplement.}

Competing interests. The author declares that there is no conflict of interest.

Acknowledgements. The author wishes to thank William Armstrong and Mauri Pelto for constructive reviews that have helped to improve both the manuscript and the tools presented in this paper. The author also wishes to thank Stephen Brough and Adam Hepburn for comments and suggestions on GEEDiT. Also acknowledged are Jack Boitier, John Cordall, Phoebe Cox, Elaine Crimmins, Ajjay Dhesi and Thomas Hutton, who extensively tested an earlier version of MaQiT as part of their undergraduate dissertations.

Edited by: David Lundbek Egholm

Reviewed by: Mauri Pelto and William Armstrong

\section{References}

Benn, D. I., Åström, J., Zwinger, T., Todd, J., Nick, F. M., Cook, S., Hulton, N. R., and Luckman, A.: Melt-under-cutting and buoyancy-driven calving from tidewater glaciers: new insights from discrete element and continuum model simulations, J. Glaciol., 63, 691-702, 2017.

Bevan, S. L., Luckman, A. J., and Murray, T.: Glacier dynamics over the last quarter of a century at Helheim, Kangerdlugssuaq 
and 14 other major Greenland outlet glaciers, The Cryosphere, 6, 923-937, https://doi.org/10.5194/tc-6-923-2012, 2012.

Björnsson, H., Pálsson, F., and Guðmundsson, S.: Jökulsárlón at Breiðamerkursanður, Vatnajökull, Iceland: 20th century changes and future outlook, Jökull, 20th century changes and future outlook, Jökull, 50, 1-18, 2001.

Bunce, C., Carr, J. R., Nienow, P. W., Ross, N., and Killick, R.: Ice front change of marine-terminating outlet glaciers in northwest and southeast Greenland during the 21 st century, J. Glaciol., 113, 2018.

Carr, J. R., Stokes, C. R., and Vieli, A.: Threefold increase in marine-terminating outlet glacier retreat rates across the Atlantic Arctic: 1992-2010, Ann. Glaciol., 58, 72-91, 2017.

Cook, A. J., Fox, A. J., Vaughan, D. G., and Ferrigno, J. G.: Retreating glacier fronts on the Antarctic Peninsula over the past half-century, Science, 308, 541-544, 2005.

D'Errico, J.: Arclength function, available at: https://uk.mathworks. com/matlabcentral/fileexchange/34871-arclength (last access: 5 July 2018) 2012a.

D'Errico, J.: interparc function, available at: https://uk.mathworks. com/matlabcentral/fileexchange/34874-interparc (last access: 5 July 2018), 2012b.

D'Errico, J.: Distance2curve function, available at: https://uk.mathworks.com/matlabcentral/fileexchange/ 34869-distance2curve, (last access: 5 July 2018), 2013.

Dugge, J.: Jdugge/xy2sn, available at: https://uk.mathworks.com/ matlabcentral/fileexchange/39796-jdugge-xy2sn (last access: 5 July 2018), 2015.

Fitzpatrick, A. A. W., Hubbard, A. L., Box, J. E., Quincey, D. J., van As, D., Mikkelsen, A. P. B., Doyle, S. H., Dow, C. F., Hasholt, B., and Jones, G. A.: A decade (2002-2012) of supraglacial lake volume estimates across Russell Glacier, West Greenland, The Cryosphere, 8, 107-121, https://doi.org/10.5194/tc-8-107-2014, 2014.

Gorelick, N., Hancher, M., Dixon, M., Ilyushchenko, S., Thau, D., and Moore, R.: Google Earth Engine: Planetary-scale geospatial analysis for everyone, Remote Sens. Environ., 202, 18-27, 2017.

Kuenzer, C., van Beijma, S., Gessner, U., and Dech, S.: Land surface dynamics and environmental challenges of the Niger Delta, Africa: Remote sensing-based analyses spanning three decades (1986-2013), Appl. Geogr., 53, 354-368, 2014.
Lea, J. M., Mair, D. W. F., and Rea, B. R.: Evaluation of existing and new methods of tracking glacier terminus change. J. Glaciol., 60, 323-332, 2014.

Lea, J.: University of Liverpool Google Earth Engine Tools/MaQiT, available at: bewww.liverpoolGEE.wordpress.com, last access: 12 July 2018.

Lynch, C., Barr, I. D., Mullan, D., and Ruffell, A.: Rapid glacial retreat on the Kamchatka Peninsula during the early 21st Century, The Cryosphere, 10, 1809-1821, https://doi.org/10.5194/tc-101809-2016, 2016.

Moon, T. and Joughin, I.: Changes in ice front position on Greenland's outlet glaciers from 1992 to 2007, J. Geophys. Res.-Earth Surf., 113, https://doi.org/10.1029/2007JF000927, 2008.

Motyka, R. J., Cassotto, R., Truffer, M., Kjeldsen, K. K., Van As, D., Korsgaard, N. J., Fahnestock, M., Howat, I., Langen, P. L., Mortensen, J., and Lennert, K. Asynchronous behavior of outlet glaciers feeding Godthåbsfjord (Nuup Kangerlua) and the triggering of Narsap Sermia's retreat in SW Greenland, J. Glaciol., 63, 288-308, 2017.

Palacios, R.: Deg2utm function, available at: https://uk.mathworks. com/matlabcentral/fileexchange/10915-deg2utm?focused= 5073379\&tab=function (last access: 5 July 2018), 2006.

Planet Labs Inc.: Planet Image Explorer, available at: https://www. planet.com/, accessed 9 February 2018.

Roelfsema, C., Kovacs, E. M., Saunders, M. I., Phinn, S., Lyons, M., and Maxwell, P.: Challenges of remote sensing for quantifying changes in large complex seagrass environments, Estuarine, Coast. Shelf Sci., 133, 161-171, 2013.

Sinergise: Sentinel Hub Earth Observation Explorer, https:// sentinel-hub.com/explore/eobrowser, last access: 9 February 2018.

Voytenko, D., Dixon, T. H., Howat, I. M., Gourmelen, N., Lembke, C., Werner, C. L., De La Peña, S., and Oddsson, B.: Multiyear observations of Breiðamerkurjökull, a marine-terminating glacier in southeastern Iceland, using terrestrial radar interferometry, J. Glaciol., 61, 42-54, 2015. 